\title{
Stimulus intensity affects pigeons' timing behavior: Implications for an internal clock model
}

\author{
DONALD M. WILKIE \\ University of British Columbia, Vancouver, British Columbia, Canada
}

\begin{abstract}
Five pigeons were trained to discriminate between 2- and 10-sec illuminations of a white light; choice of a red pecking key was correct and rewarded after presentation of the short stimulus, whereas choice of a green key was correct and rewarded after presentation of the long stimulus. On half the trials, the light was bright; on the others, it was dim. Durations of 4,6 , and $8 \mathrm{sec}$ of both dim and bright light were also presented; choices on these trials were not rewarded. The probability of the pigeons' choosing the short alternative decreased in a graded manner as duration of both bright and dim light increased from 2, to 4, to 6, to 8, and to $10 \mathrm{sec}$. However, the pigeons were more likely to choose the short alternative with longer durations of the dim light than the bright light, a result that implies that the perceived duration of a dim light was shorter than that of a bright light of equal length. One interpretation of this effect is that stimulus intensity affects the rate of the pacemaker in an internal clock mechanism subserving timing of event duration.
\end{abstract}

It has been amply demonstrated in laboratory studies that animals such as rats and pigeons can discriminate the duration of events. In a typical study using pigeons, for example, a light is turned on for either 2 or $10 \mathrm{sec}$ and followed immediately by the presentation of red and green pecking keys; pecking red is correct and rewarded after the 2-sec stimulus, whereas green is correct and rewarded after the 10-sec stimulus. Pigeons quickly learn to choose the appropriate color and, in addition, to respond to intermediate durations between 2 and $10 \mathrm{sec}$ in a graded fashion, choosing red ("short") progressively less often for durations of 4,6 , and $8 \mathrm{sec}$.

An information processing approach to animals' ability to perform such duration discriminations has been proposed by Church and his colleagues (e.g., Church, 1984). One such model, depicted in Figure 1, comprises five components: (1) a pacemaker that emits a stream of pulses; (2) a gate (switch) that is opened at the onset of the event to be timed and closed at its offset; (3) a counter that accumulates pulses; (4) two memories-a permanent ("reference") memory that stores the total number of pulses of reinforced times and a temporary ("working") memory that stores the number of pulses accumulated on a particular trial, and (5) A comparator that compares the values stored in the two memories. If the value stored in working memory is similar to the short value stored in reference memory, then a "short" choice is made; if the value stored in working memory is similar

\footnotetext{
This research was supported by the Natural Sciences and Engineering Research Council of Canada. Shayne Kardel assisted with the experiments. Send requests for reprints to Donald M. Wilkie, Department of Psychology, University of British Columbia, 2136 West Mall, Vancouver, B.C., Canada V6T 1 Y7.
}

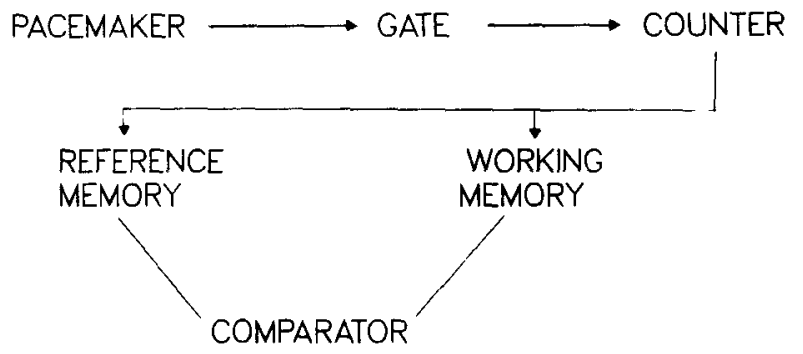

Figure 1. Schematic of internal clock model of animals' ability to discriminate duration of events.

to the long value stored in reference memory, then a "long" choice is made.

This type of model has received considerable empirical support and has recently been elaborated to account for number as well as duration discrimination (e.g., Meck \& Church, 1983).

Although in this type of model it is usually assumed that the pacemaker emits pulses at a fixed mean rate with variability, there is some evidence that its mean pulse rate may be modified by certain manipulations. Maricq, Roberts, and Church (1981) trained some of their rats to discriminate between 4- and 16-sec durations and tested the subjects with these and intermediate durations after injections of saline or the stimulant drug methamphetamine. They found that the rats classified intermediate durations as being longer after methamphetamine injections, a result that suggests that the rate of the pacemaker had increased. An example may clarify this interpretation. Assume that during training a rat's pacemaker ran at $5 \mathrm{~Hz}$. After 4- and 16-sec stimuli, the counter would read 20 
and 80 counts, respectively. During a saline test, a 6-sec stimulus would result in a counter value of 30 , a value similar to 20 and therefore likely to result in a "short" choice. If we assume that methamphetamine increases the pacemaker rate to $8 \mathrm{~Hz}$, then a 6 -sec stimulus during the drug test would result in an accumulated value of 48 , which is more similar to the stored "long" value in reference memory and consequently more likely to result in a "long" choice.

The present research provides more evidence congruent with the notion that the pacemaker rate may be manipulated. In humans, it is known that stimulus intensity affects perceived duration. For example, Goldstone, Lhamon, and Sechzer (1978) reported that more intense sounds and lights were judged to be longer than less intense presentation of these stimuli. These findings are interpretable in terms of stimulus intensity affecting the rate of the pacemaker. If more intense stimuli speed up the pacemaker-perhaps by affecting general arousal-more intense stimuli would be perceived as being longer. In the present study, pigeons were trained to discriminate short and long durations of dim and bright light and were tested with intermediate durations to determine if their tendency to classify a stimulus as short or long was different for dim and bright presentations.

\section{METHOD}

\section{Subjects}

Five King pigeons, maintained at about $90 \%$ of normal body weight, served as subjects. The pigeons were housed individually in large plastic-coated mesh cages with free access to water as well as health grit and oyster shells. The colony was maintained on a 12-h light/dark cycle; the subjects were tested 5 days a week, about $2 \mathrm{~h}$ after colony light onset. A sixth pigeon was discarded because it failed to discriminate light durations accurately.

\begin{abstract}
Apparatus
Each pigeon was tested consistently in one of five test chambers containing a houselight, grain feeder, and two pecking keys, which could be lit with red or green light. Houselight intensity was controlled by placing a resistor in series with the lamp. The "dim" and "bright" houselight intensities in each of the boxes were: Box 1, .04 and $18.5 \mathrm{~cd} / \mathrm{m}^{2}$; Box 2, .36 and $20.5 \mathrm{~cd} / \mathrm{m}^{2}$; Box $3, .45$ and $33 \mathrm{~cd} / \mathrm{m}^{2}$; Box 4, .9 and $34 \mathrm{~cd} / \mathrm{m}^{2}$; Box $5, .9$ and $14 \mathrm{~cd} / \mathrm{m}^{2}$. The red and green pecking keys were 1.25 and $.62 \mathrm{~cd} / \mathrm{m}^{2}$, respectively. All measurements were taken from a distance of 3 to $5 \mathrm{~cm}$. A Data General NOVA 3 computer and MANX state notation software (Gilbert \& Rice, 1979) controlled the experimental equipment and collected the responses.
\end{abstract}

\section{Procedure}

Pretraining. As each pigeon had had recent event-duration training with auditory stimuli, no preliminary training was needed. Each subject first was trained to discriminate between short $(2 \mathrm{sec})$ and long $(10 \mathrm{sec})$ houselight presentations. In one set of 30 sessions, bright light was used; in another, only dim light was used. In all sessions, the 20 -sec intertrial interval was spent in complete darkness. On each of the 80 trials in a session, the probability was .5 that the light presentation was short. Immediately after the light presentation, two pecking keys were lit, one with red and one with green light, with the right-left location of the colors varied randomly over trials. For some pigeons, red was designated as correct and rewarded after the short stimulus; for others, green was correct. (For convenience in exposition, we assume that red is always correct after the short stimulus.) Choice of the correct key produced 5 -sec access to mixed grain on a partial (75\%) reinforcement schedule.

Experiment proper. The pigeons received approximately 35 sessions, each comprising 80 trials. On one-half of the trials (randomly determined), the houselight was bright; on the remainder, it was dim. On a quarter of all trials (randomly determined), the light presentation was $2 \mathrm{sec}$ in duration. On another quarter, it was $10 \mathrm{sec}$. On the remaining trials, it was equally probable that the light would be 4,6 , or $8 \mathrm{sec}$ in duration. Thus, there were 10 types of trials in total-2-, 4-, 6-, 8-, and 10-sec bright signals and 2-, 4-, 6-, 8-, and 10-sec dim signals, presented in a randomized order. Correct choices on 2- and 10-sec trials of both dim and bright trials always produced 5-sec access to grain. Choices on 4-, 6-, and 8-sec trials were never rewarded.

In each session, a record was kept of the number of times the "short" choice key was selected when 2-, 4-, 6-, 8-, and 10-sec lights were presented. These values were cumulated over sessions and used to calculate the percentage of trials on which the pigeons chose the "short" alternative after durations of 2, 4, 6, 8, and $10 \mathrm{sec}$ of the light. These measures were calculated separately for bright and dim light trials.

\section{RESULTS}

As shown in Figure 2, the percentage of trials on which the pigeons chose the short alternative differed when light presentations were bright or dim. Specifically, the pigeons chose short more frequently with dim light, especially with longer durations. These observations were confirmed in a two-factor (bright vs. dim, light duration) repeated measures analysis of variance. Although the main effect of bright versus dim was only marginally significant $[F(1,4)=4.69, p=.095]$, the interaction of bright versus $\operatorname{dim}$ and light duration was highly significant $[F(4,16)=5.38, p=.006]$. As is obvious in Figure 2, the main effect of light duration was also highly significant $[F(4,16)=60.02, p<.001]$.

Post hoc Newman-Keuls tests $(p<.05)$ conducted on the bright versus $\operatorname{dim} \times$ light duration interaction revealed that the pigeons were more likely to choose short after dim light presentations of 10,8 , and $6 \mathrm{sec}$ but not after 4 or $2 \mathrm{sec}$.

The subjects' tendency to choose the shorter alternative more frequently with longer durations of the dim light did not appear to be due to a general bias toward choosing short when dim light was presented or to the fact that the dim discrimination was more difficult. In the pretraining stage, when only dim or bright light appeared in a session, the pigeons discriminated short and long presentations of dim and bright light equally well (see Table 1).

\section{DISCUSSION}

The finding that the pigeons were more likely to choose the short alternative when samples of 6,8 , or $10 \mathrm{sec}$ were dim rather than bright suggests that the perceived duration of a stimulus depends upon its intensity. It appears that dim light equal in duration to bright light appears 


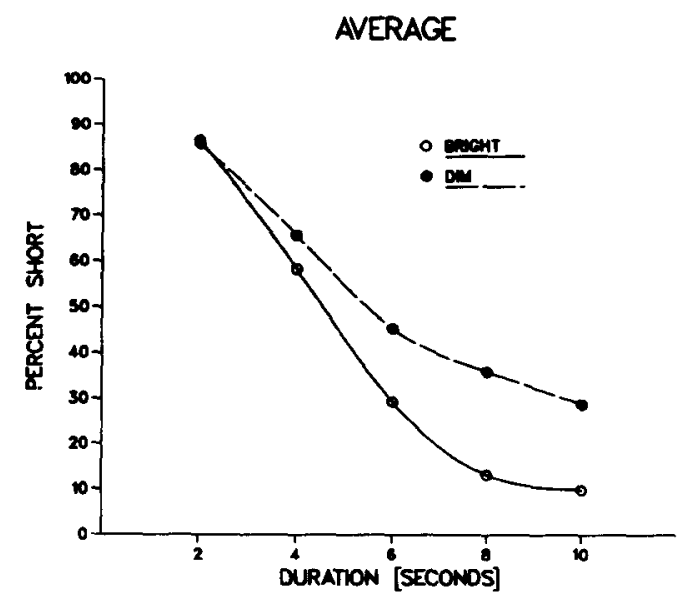

BRD 3

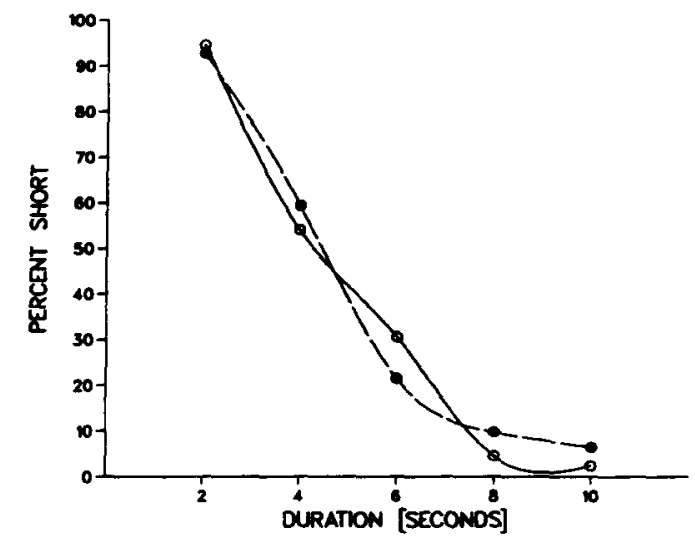

BARD 1
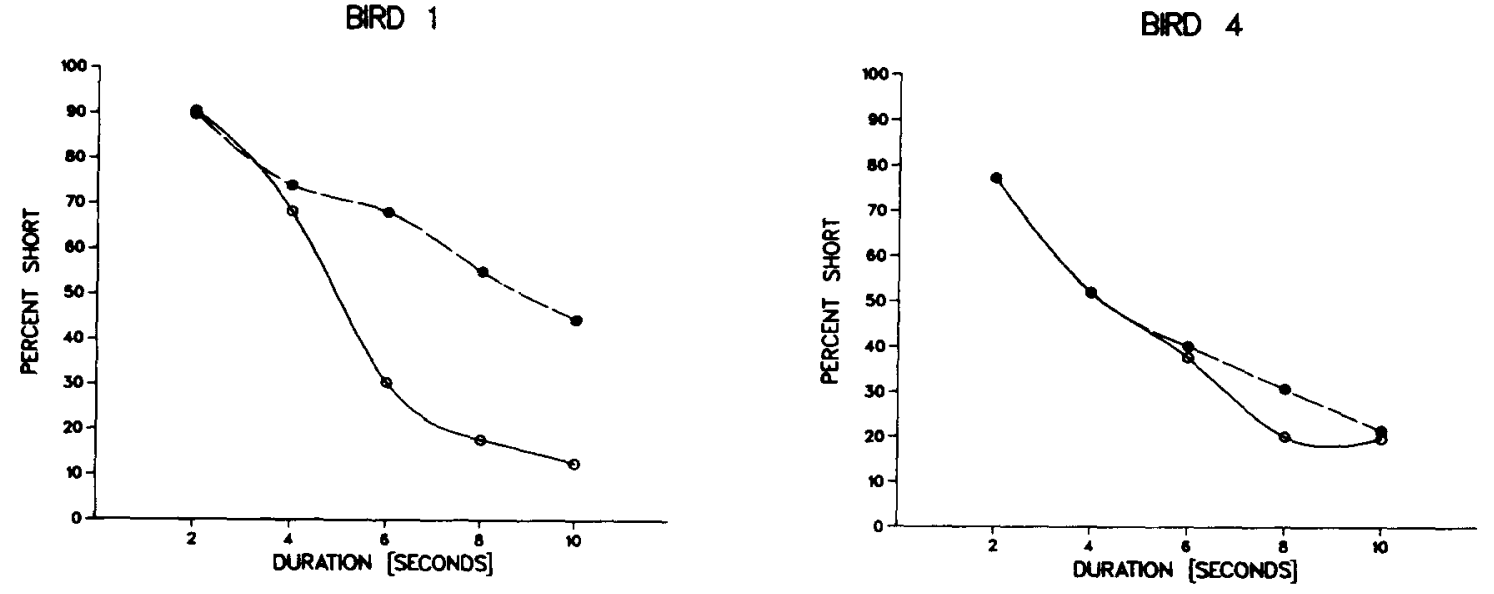

BIRD 2
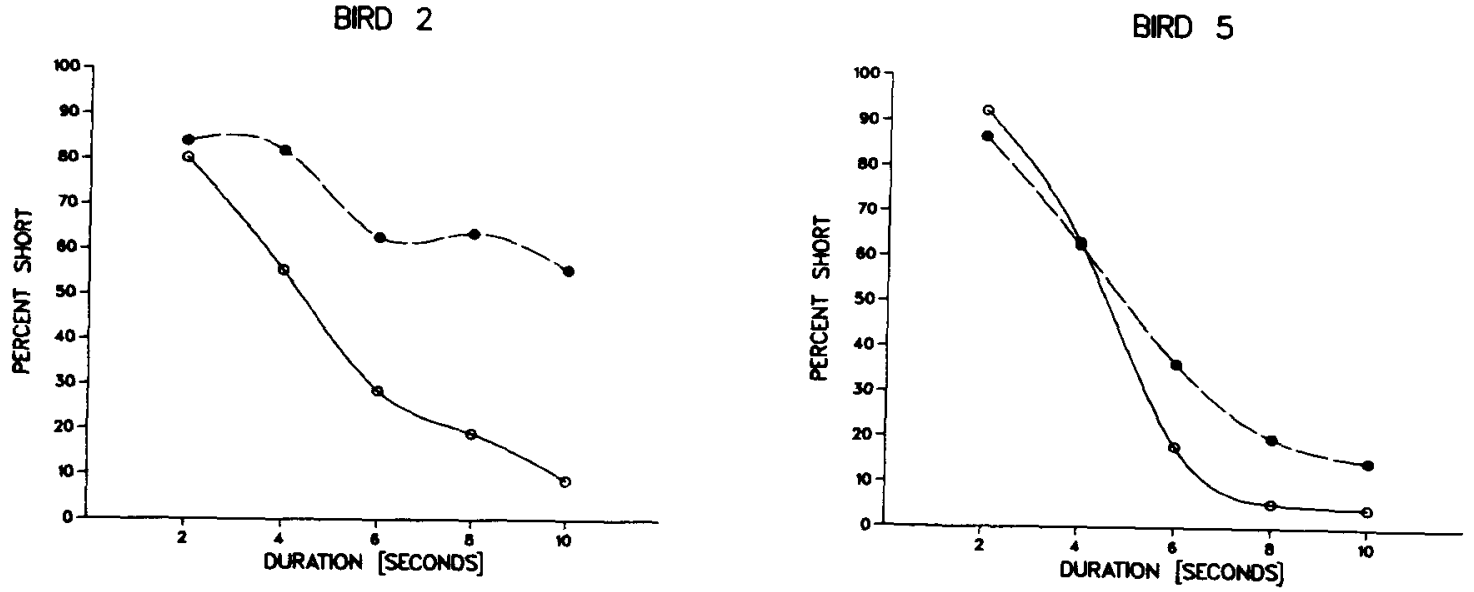

Figure 2. Percent of trials on which the pigeons chose the "short" alternative when dim $(\bullet)$ and bright $(\circ)$ lights of $2,4,6,8$, and $10 \mathrm{sec}$ were presented. Data are shown for each subject, as well as the average of all 5 pigeons. 
Table 1

Probability of Choosing the Short Alternative After 2- and 10-sec Presentations of Dim and Bright Houselights During Preliminary Training

\begin{tabular}{cccccc}
\hline & \multicolumn{2}{c}{ Dim } & & \multicolumn{2}{c}{ Bright } \\
\cline { 2 - 3 } \cline { 5 - 6 } Bird & $2 \mathrm{sec}$ & $10 \mathrm{sec}$ & & $2 \mathrm{sec}$ & $10 \mathrm{sec}$ \\
\hline 1 & .77 & .08 & .79 & .16 \\
2 & .97 & .02 & & .97 & .02 \\
3 & .90 & .06 & .84 & .01 \\
4 & .80 & .14 & .80 & .17 \\
5 & .76 & .08 & & .94 & .07 \\
Average & .84 & .08 & .87 & .09 \\
\hline
\end{tabular}

Note-Data are averages across last 3 of 30 sessions of training with both dim and bright light.

shorter. One interpretation of this effect is that stimulus intensity affects the pacemaker component of the internal clock mechanism subserving event duration discrimination. Specifically, it may be the case that the pacemaker puts out pulses at a higher mean rate when stimuli are more intense. As a consequence, cumulated clock ticks are fewer with dim than with bright stimuli. Because the number of ticks stored in the working memory after a dim presentation are more similar to the "short" value stored in reference memory, short choices are more probable.

This interpretation parallels the one offered by Marica et al. (1981) to account for the effects of methamphetamine on event-duration discrimination. Both their results and the present ones imply that an animal's internal clock-specifically its pacemaker's average rate-is not absolutely fixed but can be varied. Although admittedly speculative, it seems possible that the drug and lightintensity effects might both be mediated by a state of arousal that affects the pacemaker rate.

It is interesting to note that the pigeons discriminated short and long durations of dim and bright lights equally well when only one stimulus intensity was presented in a session. In the preliminary sessions, there was no tendency to choose the short alternative more often when the light was dim than when it was short. This difference implies that reference memory played a critical role in the results obtained in sessions when both dim and bright lights appeared. When only one intensity appears in a set of sessions (as was the case in preliminary training), the clock values stored in reference memory mirror the prevailing pacemaker rate. For example, in a set of sessions with dim light, the reference memory value for the short stimulus might be 4 , assuming a pacemaker rate of $2 \mathrm{~Hz}$, whereas in a set of sessions with bright light, the reference value for the same short stimulus might be 10 , assuming a pacemaker rate of $5 \mathrm{~Hz}$. Under these conditions, the values stored in working memory also reflect the prevailing pacemaker rate and subjects classify durations accurately. The situation is different, however, when both dim and bright stimuli appear in the same session. Because reference memory contains only one pair of values (theoretically it could be the pacemaker's values for either a dim or a bright stimulus) and the subject must choose short or long on the basis of the comparison of working and reference memory value, subjects' choices are biased in one direction or another. An example will make this point clearer. Assume that a subject stores the bright counts in reference memory, values of 10 and 50 for short and long signals, assuming a $5-\mathrm{Hz}$ pacemaker. Longer durations of bright light will produce working memory counts of 20 (4-sec signal), 30 (6-sec signal), and so forth, and a decreased probability of choosing short. With dim stimuli of corresponding durations, working memory counts will be 8,12 , and so forth, assuming a 2-Hz pacemaker. Because these working memory values resemble the short bright reference memory value (10), the subjects will tend to choose short.

Theoretically, subjects could store either bright or dim counts in reference memory and treat bright durations as being longer (if the dim value was stored in reference memory) or dim value as being shorter (if the bright value was stored in reference memory). Our results suggest that the latter occurred in our subjects-longer durations of dim stimuli were classified as shorter. At present, we are unable to offer a good explanation for the apparent bias for the storage of bright values in reference memory. However, experience may play a role as illumination in the room in which the pigeons lived was quite high $\left(60 \mathrm{~cd} / \mathrm{m}^{2}\right)$. It is interesting to note that Meck (1984) has found a bias between light and sound signals which may also vary on an intensity continuum.

It should be acknowledged that it is theoretically possible that subjects might, after extended training-after much more training than our subjects received-store both short and long counts of both dim and bright signals in reference memory (along with the corresponding response or decision rules). In that case, the percentage of trials on which subjects chose the short alternative would be no different on dim and bright trials.

Given that the internal clock models generally comprise five or so components, some comments are warranted concerning our suggestion that intensity seems to affect only one component in the model, namely the pacemaker. Although intensity could theoretically affect any of the constituent components (e.g., counter, memories), it is not intuitively obvious how intensity would affect something like a counter or how any such effect would be manifested in dim signals' being perceived as being shorter. A more reasonable possibility might be that intensity affects the switching process, so that timing begins after a longer latency on dim signal trials. However, our results seem to be incompatible with this mechanism. If timing began more slowly with dim signals, this would have the effect of shortening all dim signals $(2,4,6,8$, and $10 \mathrm{sec}$ ) by a fixed amount. Thus, the function relating short choices to duration should be shifted upward by a constant amount from the bright signal function. In other words, the dim and bright functions should be parallel. Our data (Figure 2), however, show instead a clear divergence between the dim and bright functions. 


\section{REFERENCES}

Church, R. M. (1984) Properties of the internal clock. In J. Gibbon \& L. Allan (Eds.), Annals of the New York Academy of Sciences: Timing and time perception (Vol. 423, pp. 566-582). New York: New York Academy of Sciences.

GILbERT, S. G., \& RICE, D. C. (1979). NOVA SKED II: A behavioral notation language utilizing the Data General Corporation real-time operating system. Behavior Research Methods \& Instrumentation, 11, 71-73.

Goldstone, S., Lhamon, W. T., \& Sechzer, J. (1978). Light intensity and judged duration. Bulletin of the Psychonomic Society, 12, 83-84.

MaricQ, A. V., Roberts, S., \& ChURCh, R. M. (1981). Methampheta- mine and time estimation. Journal of Experimental Psychology: Animal Behavior Processes, 7, 18-30.

MeCK, W. H. (1984). Attentional bias between modalities: Effect on the internal clock, memory, and decision stages used in animal time discrimination. In J. Gibbon \& L. Allan (Eds.), Annals of the New York Academy of Sciences: Timing and time perception (Vol. 423, pp. 528-545). New York: New York Academy of Sciences.

MeCK, W. H., CHURCH, R. M. (1983). A mode control model of counting and timing processes. Journal of Experimental Psychology: Animal Behavior Processes, 9, 320-334.

(Manuscript received February 13, 1986; revision accepted for publication September 10, 1986.) 